J. DIFFERENTIAL GEOMETRY

77 (2007) 353-384

\title{
LARGE TIME BEHAVIOR OF HEAT KERNELS ON FORMS
}

\author{
Thierry Coulhon \& Qi S. Zhang
}

\begin{abstract}
We derive large time upper bounds for heat kernels on vector bundles of differential forms on a class of non-compact Riemannian manifolds under certain curvature conditions.
\end{abstract}

\section{Introduction}

The goal of the present paper is to establish large time, pointwise bounds for the heat kernel on the vector bundles of forms on some noncompact manifolds.

Information on large time behavior of heat kernels on forms usually leads to interesting analytical and topological information on the manifolds. In fact, a heat kernel on forms contains much more information on the interplay between analysis, geometry, and topology than that on functions. So far much effort has been spent on the study of short time and long time behavior of heat kernel on forms, in the case of closed manifolds; see for instance [3], [30]. By contrast, the present paper is to our knowledge the first one to offer estimates for the heat kernel on one-forms on a class of non-compact Riemannian manifolds with a meaningful contents for large time, i.e., without an increasing exponential factor (see for instance [27], [5]).

Let $M$ be a complete connected Riemannian manifold. Denote by $d(x, y)$ the geodesic distance between two points $x, y \in M$, and by $B(x, r)$ the open ball of center $x \in M$ and radius $r>0$. Let $\mu$ be the Riemannian measure; denote also by $|\Omega|$ the measure $\mu(\Omega)$ of a mesurable subset $\Omega$ of $M$. Denote by $\Delta$ the (non-negative) LaplaceBeltrami operator on functions. The heat semigroup on functions $e^{-t \Delta}$ will also be denoted by $P_{t}$, and the corresponding heat kernel by $p_{t}(x, y)$, $t>0, x, y \in M$. We will use $\vec{\Delta}$ to denote the Hodge Laplacian on forms. The heat semigroup on forms $e^{-t \vec{\Delta}}$ will also be denoted by $\vec{P}_{t}$, and the corresponding heat kernel by $\vec{p}_{t}(x, y), t>0, x, y \in M$.

TC's research was partially supported by the European Commission (IHP Network "Harmonic Analysis and Related Problems" 2002-2006, Contract HPRN-CT-200100273-HARP).

Received 06/07/2005. 
The main question we shall address below is the following:

Given an upper estimate for the heat kernel on functions, under which additional assumptions can one deduce an upper bound for the heat kernel on forms?

We shall consider in particular the case where $M$ has the so-called volume doubling property and the heat kernel on functions satisfies a Gaussian upper estimate, that is,

$$
p_{t}(x, y) \leq \frac{C}{|B(x, \sqrt{t})|} \exp \left(-c d^{2}(x, y) / t\right), \forall x, y \in M, t>0,
$$

for some $C, c>0$.

For instance, when $M$ has non-negative Ricci curvature, it was proved in $[\mathbf{2 6}]$ that $p_{t}$ satisfies a Gaussian upper estimate, and in that case, the answer to the above question for 1-forms is straightforward by the semigroup domination theory (see (1.3) below, and also e.g., [22], [23], $[\mathbf{1 5}],[\mathbf{2 9}])$ : the heat kernel on 1 -forms is also bounded from above by a Gaussian.

The following simple example shows that this may be false in general. Let $M$ be the connected sum of two copies of $\mathbb{R}^{n}, n \geq 3$. It is known that the heat kernel on functions has a Gaussian upper bound (see [2]). If the heat kernel on 1-forms $\vec{p}_{t}$ also had a Gaussian upper bound, then by $[\mathbf{1 1}]$, pp. 1740-1741, it would follow that

$$
\left|\nabla p_{t}(x, y)\right| \leq \frac{C^{\prime}}{\sqrt{t}|B(x, \sqrt{t})|} \exp \left(-c^{\prime} d^{2}(x, y) / t\right), \forall x, y \in M, t>0 .
$$

A classical argument shows that $p_{t}$ would then be bounded below by a Gaussian (see for instance [26]). This is false, as was noticed in [2]. Another reason why (1.1) cannot hold in this case is the existence of non-trivial $L^{2}$ harmonic forms. See also [10] for more on this example.

Another case where the behaviour of the heat kernel on 1 -forms is well understood is the case of the Heisenberg group and more generally stratified Lie groups, see [31], [32].

In the present paper, we are going to see that if the negative part of the Ricci curvature is small enough in some sense, then the upper bound on the heat kernel on 1-forms differs from that on the heat kernel on functions at most by a certain power of time $t$. One can state similar results for higher degree forms by replacing in the assumptions the Ricci curvature by a suitable curvature operator (see $[\mathbf{1 6}]$ ). For convenience, we shall, however, formulate our assumptions and results in the case of 1 -forms. We leave the formulation of the general case to the reader.

In this article, all Riemannian manifolds under consideration will be complete non-compact. Let us lay out some basic assumptions to be used below. 
Assumption (A). $M$ satisfies the volume doubling property:

$$
|B(x, 2 r)| \leq C|B(x, r)|
$$

for some $C>0$ and all $x \in M, r>0$.

Assumption (B). The heat kernel $p_{t}(x, y)$ on functions satisfies an on-diagonal upper bound:

$$
p_{t}(x, x) \leq \frac{C}{|B(x, \sqrt{t})|},
$$

for some $C>0$, and all $x \in M, t>0$.

It was proved in $[\mathbf{1 7}]$ that Assumptions (A) and (B) together are equivalent to the following relative Faber-Krahn inequality:

For some $c, \nu>0$, all $x \in M, r>0$, and every non-empty subset $\Omega \subset B(x, r)$,

$$
\lambda_{1}(\Omega) \geq \frac{c}{r^{2}}\left(\frac{|B(x, r)|}{|\Omega|}\right)^{2 / \nu} .
$$

Here $\lambda_{1}(\Omega)$ is the first Dirichlet eigenvalue of $\Omega$.

Note that Assumption (A) is equivalent to

$$
\frac{|B(x, s)|}{|B(x, r)|} \leq C\left(\frac{s}{r}\right)^{\nu}
$$

for some $C, \nu>0$, all $s>r>0, x \in M$, and we shall often use this formulation.

Note finally that (A) and (B) imply the Gaussian upper bound:

$$
p_{t}(x, y) \leq \frac{C}{|B(x, \sqrt{t})|} \exp \left(-c d^{2}(x, y) / t\right),
$$

for some $C, c>0$, and all $x, y \in M, t>0$ (see [20], Theorem 1.1).

Assumption (C). The Ricci curvature is bounded from below by a negative constant.

It follows from Assumption (C) and Bishop's comparison theorem that there exists $C>0$ such that $|B(x, 1)| \leq C$ for all $x \in M$. We shall often also need the opposite inequality.

Assumption (D). Non-collapsing of the volume of balls: there exists $c>0$ such that $|B(x, 1)| \geq c$ for all $x \in M$.

Note that assumption (D) is satisfied if the injectivity radius is bounded from below by a positive constant and the sectional curvature is bounded from above.

It is well-known that to estimate the heat kernel acting on one-forms, it is enough to estimate the kernel of a certain Schrödinger semigroup acting on functions, whose potential is the negative part of the Ricci curvature. 
Indeed, Bochner's formula states

$$
\vec{\Delta}=D^{*} D+\text { Ric. }
$$

Here $D$ is the covariant derivative on 1 -forms and Ric is the Ricci curvature.

Let $\lambda=\lambda(x)$ be the lowest eigenvalue of Ric $(x), x \in M$. We will use the notation

$$
V(x)=\lambda^{-}(x)=(|\lambda(x)|-\lambda(x)) / 2 .
$$

Let $P_{t}^{V}$ be the semigroup $e^{-t(\Delta-V)}$. Under Assumption (C), $P_{t}^{V}$ has a kernel which we shall denote by $p_{t}^{V}(x, y)$.

Let us recall the semi-group domination property, which was proved in $[\mathbf{2 3}]$ :

$$
\left|\vec{p}_{t}(x, y)\right| \leq p_{t}^{V}(x, y)
$$

for all $x, y \in M$ and $t>0$. Here $\vec{p}_{t}(x, y)$ is a linear operator between the cotangent spaces $T_{y}^{*} M$ and $T_{x}^{*} M$, and $\left|\vec{p}_{t}(x, y)\right|$ denotes its operator norm with respect to the Riemannian metrics.

We can now introduce one of our main curvature assumptions. An important property of the Hodge Laplacian $\vec{\Delta}$ is that it is a nonnegative operator (as a consequence, $\vec{P}_{t}$ is contractive on $L^{2}\left(M, T^{*} M\right)$ ). This means that, for every smooth compactly supported 1-form $\phi$,

$$
\int_{M}-\operatorname{Ric}(\phi(x), \phi(x)) d \mu(x) \leq \int_{M}|D \phi(x)|^{2} d \mu(x) .
$$

Now, by the Kato inequality

$$
|\nabla| \phi|| \leq|D \phi|
$$

and the fact that by definition

$$
\operatorname{Ric}(\phi(x), \phi(x)) \geq-V(x)|\phi(x)|^{2},
$$

we see that condition (1.4) is implied by

$$
\int_{M} V(x) f^{2}(x) d \mu(x) \leq \int_{M}|\nabla f(x)|^{2} d \mu(x), \forall f \in C_{0}^{\infty}(M),
$$

which means that $\Delta-V$ is a positive operator on $L^{2}(M)$.

We shall say that $\Delta-V$ is strongly positive (strongly subcritical in the sense of [14]) if it satisfies the following stronger condition: there exists $A<1$ such that, for all $f \in C_{0}^{\infty}(M)$,

$$
\int_{M} V f^{2} d \mu \leq A \int_{M}|\nabla f|^{2} d \mu .
$$

The above condition sometimes is referred to as the form boundedness condition, which has its origin in the Hardy inequality: for $f \in C_{0}^{\infty}\left(\mathbb{R}^{n}\right)$, $n \geq 3$,

$$
\frac{(n-2)^{2}}{4} \int_{\mathbb{R}^{n}} \frac{f^{2}(x)}{|x|^{2}} d x \leq \int_{\mathbb{R}^{n}}|\nabla f(x)|^{2} d x .
$$


For generalizations of the above inequality to the manifold case, see [4].

Example. If the manifold $M$ satisfies the Euclidean Sobolev inequality of dimension $n$

$$
\left(\int_{M}|f|^{2 n /(n-2)} d \mu\right)^{\frac{n-2}{n}} \leq C \int_{M}|\nabla f|^{2} d \mu
$$

for all $f \in C_{0}^{\infty}(M)$, for some $n>2$, and if $V \in L^{n / 2}(M)$ with sufficiently small norm, then it is easy to see by using Hölder's inequality that (1.6) holds.

Let us now summarize our results. Under Assumptions (A) to (D), the function $V$, the negative part of the lowest eigenvalue of the Ricci curvature, largely determines the upper bound of heat hernel on 1forms. If $V$ is sufficiently small in a certain integral sense, then $\vec{p}_{t}$ has a Gaussian upper bound, which has important consequences in terms of $L^{p}$ boundedness of the Riesz transform. This is explained in Section 3. Otherwise, the upper bound for $\overrightarrow{p_{t}}$ is a Gaussian times a suitable power of time $t$, provided that the operator $\Delta-V$ is strongly positive. The proof of this fact is contained in Sections 2.1, 2.2, 2.3. We also consider the case where the Ricci curvature is nonnegative outside of a compact set. Without any other assumptions on the Ricci curvature, there may be $L^{2}$ harmonic forms; therefore one cannot expect a decay with respect to time in general, but we show that $\overrightarrow{p_{t}}$ is bounded by a Gaussian plus the product of the Green's function of the Laplacian in both variables. This is the subject of Section 4. Finally, we treat in Section 5 the case where the heat kernel on functions has an arbitrary uniform decay.

Acknowledgement. The second author acknowledges the support of the University of Cergy-Pontoise during the preparation of this paper. Both authors thank Gilles Carron, El-Maati Ouhabaz and Adam Sikora for nice remarks on the manuscript.

\section{Bounds for the heat kernel on forms and strong positivity}

Our aim in this section is the following result.

Theorem 2.1. Suppose $M$ satisfies Assumptions (A), (B), (C), and (D), and that the operator $\Delta-V$ is strongly positive with constant $A$. Suppose in addition that $V \in L^{p}(M, \mu)$ for some $p \in[1,+\infty)$. Then, if $p=1$, for any $c \in(0,1 / 4)$, and any $\varepsilon>0$, there exists $C>0$ such that $\left|\vec{p}_{t}(x, y)\right| \leq C \min \left\{\frac{t^{(1+\varepsilon) A}}{|B(x, \sqrt{t})|}, 1\right\} \exp \left(-c d^{2}(x, y) / t\right), \forall x, y \in M, t \geq 1$;

if $p \in(1,2)$, for any $c \in(0,1 / 4)$, there exists $C, \varepsilon>0$ such that $\left|\vec{p}_{t}(x, y)\right| \leq C \min \left\{\frac{t^{(p-\varepsilon) A}}{|B(x, \sqrt{t})|}, 1\right\} \exp \left(-c d^{2}(x, y) / t\right), \forall x, y \in M, t \geq 1$; 
if $p \geq 2$, for any $c \in(0,1 / 4)$ and any $\varepsilon>0$, there exists $C$ such that $\left|\vec{p}_{t}(x, y)\right| \leq C \min \left\{\frac{t^{(p-1+\varepsilon) A}}{|B(x, \sqrt{t})|}, 1\right\} \exp \left(-c d^{2}(x, y) / t\right), \forall x, y \in M, t \geq 1$.

Remark. Using Assumption (C) and the Gaussian bound on $p_{t}$, one easily obtains the following small time estimate

$$
\left|\vec{p}_{t}(x, y)\right| \leq p_{t}^{V}(x, y) \leq \frac{C_{t_{0}}}{|B(x, \sqrt{t})|} \exp \left(-c d^{2}(x, y) / t\right)
$$

for all $x, y \in M$ and $0<t<t_{0}$.

Thanks to the domination property (1.3), Theorem 2.1 is a consequence of the following statement, which is of independent interest. Here $V$ is any positive bounded potential.

Theorem 2.2. Suppose $M$ satisfies Assumptions (A), (B), (C), and (D), and that the operator $\Delta-V$ is strongly positive with constant $A$. Suppose in addition that $V \in L^{p}(M, \mu)$ for some $p \in[1,+\infty)$. Then, if $p=1$, for any $c \in(0,1 / 4)$ and any $\varepsilon>0$, there exists $C>0$ such that

$p_{t}^{V}(x, y) \leq C \min \left\{\frac{t^{(1+\varepsilon) A}}{|B(x, \sqrt{t})|}, 1\right\} \exp \left(-c d^{2}(x, y) / t\right), \forall x, y \in M, t \geq 1$;

if $p \in(1,2)$, for any $c \in(0,1 / 4)$, there exists $C, \varepsilon>0$ such that

$p_{t}^{V}(x, y) \leq C \min \left\{\frac{t^{(p-\varepsilon) A}}{|B(x, \sqrt{t})|}, 1\right\} \exp \left(-c d^{2}(x, y) / t\right), \forall x, y \in M, t \geq 1$;

if $p \geq 2$, for any $c \in(0,1 / 4)$ and any $\varepsilon>0$, there exists $C$ such that

$p_{t}^{V}(x, y) \leq C \min \left\{\frac{t^{(p-1+\varepsilon) A}}{|B(x, \sqrt{t})|}, 1\right\} \exp \left(-c d^{2}(x, y) / t\right), \forall x, y \in M, t \geq 1$.

We would like to mention a number of previous papers that deal with Schrödinger heat kernels on manifolds. In the paper [26], a fundamental gradient estimate was derived for the heat kernel. As far as long time behavior is concerned, the emphasis is on the case without potential and nonnegative Ricci curvature. The paper [38] studied Schrödinger heat kernels with singular oscillating potentials. The papers $[\mathbf{4 2}, \mathbf{4 3}]$ established long time behavior for Schrödinger heat kernels on manifolds with nonnegative Ricci curvature for potentials essentially behaving as negative powers of the distance function. The case of potentials with polynomial growth and magnetic field is considered in [33], on Lie groups with polynomial growth. In [24], and also in [21], Theorem 1.2, an estimate of the number of the negative of eigenvalues of $\Delta-V$ is deduced from some information of the decay of the heat kernel associated with $\Delta$, which is somewhat close in spirit to our results.

Here is the plan of the proof of Theorem 2.2. 
In Section 2.1, we show that, given the upper bound on $p_{t}$ and the strong positivity of $\Delta-V$, a pointwise upper bound on $p_{t}^{V}$ follows from an adaptation of the Nash method due to Grigor'yan, provided some $L^{1}$ to $L^{1}$ estimates for $P_{t}^{V}$ are available. In Section 2.2, we prove such estimates under the other assumptions of Theorem 2.2, and we finish the proof.

2.1. Pointwise estimates. Let us first prove the following preliminary estimate (see [13] for a similar estimate under different assumptions). We owe to Gilles Carron a way to write the proof below, which does not require strong positivity of $\Delta-V$, but only positivity. Note that a similar proof yields directly the same estimate for $\left|\vec{p}_{t}(x, y)\right|$ instead of $p_{t}^{V}(x, y)$, without any assumption other than $(\mathrm{C})$ (see Step 1 in the proof of Theorem 4.1 below).

Proposition 2.1. Suppose $M$ satisfies Assumption (C), and that the operator $\Delta-V$ is positive. Then there exist $C, c>0$ such that

$$
p_{t}^{V}(x, y) \leq C \exp \left(-c d^{2}(x, y) / t\right), \forall x, y \in M, t \geq 1 .
$$

Proof. This can be proven by a standard method of using exponential weights as in $[\mathbf{1 9}]$. An alternative way is to use wave equation method as in $[\mathbf{6}]$ or $[\mathbf{3 4}]$. Here we choose the first approach and we give the proof for the sake of completeness.

Fix $y$ and write

$$
\begin{gathered}
u(x, t)=p_{t}^{V}(x, y), \\
I(t)=\int_{M} u^{2}(x, t) w(x, t) d \mu(x)
\end{gathered}
$$

where $w(x, t)=e^{\frac{d^{2}(x, y)}{D t}}$ for some $D>0$ to be chosen later.

One has

$$
\begin{aligned}
\frac{d}{d t} I(t) & =\frac{d}{d t} \int_{M} u^{2} w d \mu \\
& =2 \int_{M} u w(-\Delta u+V u) d \mu-\int_{M} \frac{u^{2} w d^{2}(x, y)}{D t^{2}} d \mu(x) .
\end{aligned}
$$

This implies, after integration by parts,

$$
\begin{aligned}
\frac{d}{d t} I(t)= & -2 \int_{M}|\nabla u|^{2} w d \mu-2 \int_{M} u \nabla u \cdot \nabla w d \mu \\
& +2 \int_{M} V(u \sqrt{w})^{2} d \mu-\int_{M} \frac{u^{2} w d^{2}(x, y)}{D t^{2}} d \mu(x) .
\end{aligned}
$$

Then, condition (1.5) yields

$$
\begin{aligned}
\frac{d}{d t} I(t) \leq & -2 \int_{M}|\nabla u|^{2} w d \mu-2 \int_{M} u \nabla u \cdot \nabla w d \mu \\
& +2 \int_{M}|\nabla(u \sqrt{w})|^{2} d \mu-\int_{M} \frac{u^{2} w d^{2}(x, y)}{D t^{2}} d \mu(x) .
\end{aligned}
$$


Now

$$
\begin{aligned}
|\nabla(u \sqrt{w})|^{2} & =|\nabla u|^{2} w+u \nabla u \cdot \nabla w+\frac{u^{2}|\nabla w|^{2}}{4 w} \\
& =|\nabla u|^{2} w+u \nabla u \cdot \nabla w+\frac{u^{2} w d^{2}(x, y)}{D^{2} t^{2}},
\end{aligned}
$$

thus

$$
\begin{aligned}
\frac{d}{d t} I(t) & \leq \int_{M} \frac{u^{2} w d^{2}(x, y)}{D^{2} t^{2}} d \mu(x)-\int_{M} \frac{u^{2} w d^{2}(x, y)}{D t^{2}} d \mu(x) \\
& \leq-\int_{M} \frac{u^{2} w d^{2}(x, y)}{2 D t^{2}} d \mu(x)
\end{aligned}
$$

provided $D$ is chosen large enough. As a consequence,

In particular,

$$
\frac{d}{d t} I(t) \leq 0, \forall t>0
$$

$$
\int_{M}\left(p_{t}^{V}(x, y)\right)^{2} e^{\frac{d^{2}(x, y)}{D t}} d \mu(x) \leq \int_{M}\left(p_{1}^{V}(x, y)\right)^{2} e^{\frac{d^{2}(x, y)}{D}} d \mu(x)
$$

when $t \geq 1$. By (2.1) and the small time Gaussian estimate for $p_{t}$ under Assumption (C) (see [26]),

$$
p_{1}^{V}(x, y) \leq \frac{C}{|B(x, 1)|} e^{-c d^{2}(x, y)} .
$$

Using the well-known fact that a manifold satisfying $(\mathrm{C})$ has at most exponential volume growth around any point, we have, for $D>0$ large enough,

$$
\int_{M}\left(p_{1}^{V}(x, y)\right)^{2} e^{\frac{d^{2}(x, y)}{D}} d \mu(x) \leq C
$$

hence,

$$
\int_{M}\left(p_{t}^{V}(x, y)\right)^{2} e^{\frac{d^{2}(x, y)}{D t}} d \mu(x) \leq C, \quad t \geq 1 .
$$

Next, using the semigroup property

$$
\begin{aligned}
p_{2 t}^{V}(x, y)= & \int_{M} p_{t}^{V}(x, z) p_{t}^{V}(z, y) d \mu(z) \\
= & \int_{M} e^{\frac{d^{2}(x, z)}{2 D t}} p_{t}^{V}(x, z) e^{\frac{d^{2}(z, y)}{2 D t}} p_{t}^{V}(z, y) e^{-\frac{d^{2}(x, z)}{2 D t}-\frac{d^{2}(z, y)}{2 D t}} d \mu(z) \\
\leq & e^{-\frac{d^{2}(x, y)}{4 D t}}\left[\int_{M} e^{\frac{d^{2}(x, z)}{D t}}\left(p_{t}^{V}(x, z)\right)^{2} d \mu(z)\right]^{1 / 2} \\
& \cdot\left[\int_{M} e^{\frac{d^{2}(y, z)}{D t}}\left(p_{t}^{V}(y, z)\right)^{2} d \mu(z)\right]^{1 / 2} \cdot
\end{aligned}
$$

Hence,

$$
p_{t}^{V}(x, y) \leq C e^{-c d^{2}(x, y) / t}, \quad t \geq 2 .
$$


This proves the claim.

q.e.d.

We can now state our main technical result, which is an adaptation of the Nash method to the case where the semigroup under consideration is not necessarily contractive on $L^{1}$. The argument is based on the one in the proof of [18], Theorem 1.1 (see also [12], Proposition 8.1), with certain modification and localization. If $T$ is an operator from $L^{p_{1}}$ to $L^{p_{2}}$, then $\|T\|_{p_{1}, p_{2}}$ will denote the operator norm $\sup _{f \in L^{p_{1}} \backslash\{0\}} \frac{\|T f\|_{p_{2}}}{\|f\|_{p_{1}}}$.

Proposition 2.2. Let $M$ satisfy Assumptions (A), (B), (C), and (D). Suppose that $\Delta-V$ is strongly positive and that there exists a non-decreasing function $F$ such that

$$
\left\|P_{t}^{V}\right\|_{1,1} \leq F(t), t \geq 1 .
$$

Then there exists $C>0$ such that

$$
p_{t}^{V}(x, x) \leq C \frac{F^{2}(t)[\ln (e+t F(t))]^{\nu / 2}}{|B(x, \sqrt{t})|},
$$

for all $x \in M$ and $t \geq 1$, where $\nu>0$ is the constant from (1.2). If, in addition, $F$ satisfies

$$
F(2 t) \leq C F(t), \forall t \geq 1,
$$

for some $C>0$, then for any $c \in(0,1 / 4)$, there exists $C>0$ such that

$$
p_{t}^{V}(x, y) \leq C \frac{F^{2}(t)[\ln (e+t)]^{\nu / 2}}{|B(x, \sqrt{t})|} \exp \left(-c d^{2}(x, y) / t\right)
$$

for all $x, y \in M$ and $t \geq 1$.

Proof. Fix $x_{0} \in M$, write $u(x, t)=p_{t}^{V}\left(x, x_{0}\right), t>0, x \in M$, and set

$$
I(t)=\int_{M} u^{2}(x, t) d \mu(x)=p_{2 t}^{V}\left(x_{0}, x_{0}\right) .
$$

Then

$$
\begin{aligned}
I^{\prime}(t) & =-2 \int_{M} u(x, t)(\Delta u-V u)(x, t) d \mu(x) \\
& =-2 \int_{M}|\nabla u|^{2} d \mu+2 \int_{M} V u^{2} d \mu .
\end{aligned}
$$

Using assumption (1.6), we have

$$
I^{\prime}(t) \leq-2(1-A) \int_{M}|\nabla u(x, t)|^{2} d \mu(x) .
$$

Since, for any $s>0$,

$$
u^{2} \leq(u-s)_{+}^{2}+2 s u,
$$

we can write

$$
I(t) \leq \int_{\{x \mid u(x, t)>s\}}(u(x, t)-s)^{2} d \mu(x)+2 s \int_{M} u(x, t) d \mu(x) .
$$


By assumption (2.3) and the definition of $\lambda_{1}$, this yields

$$
I(t) \leq \frac{\int_{\{x \mid u(x, t)>s\}}|\nabla(u(x, t)-s)|^{2} d \mu(x)}{\lambda_{1}(\{x \mid u(x, t)>s\})}+2 s F(t),
$$

hence

$$
I(t) \leq \frac{\int_{\{x \mid u(x, t)>s\}}|\nabla u(x, t)|^{2} d \mu(x)}{\lambda_{1}(\{x \mid u(x, t)>s\})}+2 s F(t) .
$$

The bound (2.2) yields

$$
u(x, t) \leq C_{1} e^{-c_{2} d^{2}\left(x, x_{0}\right) / t},
$$

therefore

$$
\begin{aligned}
& \{x \mid u(x, t)>s\} \subset\left\{x \mid e^{-c_{2} d^{2}\left(x, x_{0}\right) / t}>s / C_{1}\right\} \\
& =\left\{x \mid d^{2}\left(x, x_{0}\right)<c_{2}^{-1} t \ln \left(C_{1} / s\right)\right\} .
\end{aligned}
$$

Thus

$$
\{x \mid u(x, t)>s\} \subset B\left(x_{0}, r\right),
$$

where

$$
r=\sqrt{c_{2}^{-1} t\left(\left|\ln \left(C_{1} / s\right)\right|+1\right)}
$$

(we choose to take $r \geq c \sqrt{t}$ for later convenience). According to (FK), we have

$$
\lambda_{1}(\{x \mid u(x, t)>s\}) \geq \frac{c}{r^{2}}\left(\frac{\left|B\left(x_{0}, r\right)\right|}{|\{x \mid u(x, t)>s\}|}\right)^{2 / \nu} .
$$

On the other hand,

$$
|\{x \mid u(x, t)>s\}| \leq s^{-1} \int_{M} u(x, t) d \mu(x) \leq s^{-1} F(t) .
$$

Therefore

$$
\lambda_{1}(\{x \mid u(x, t)>s\}) \geq \frac{c}{r^{2}}\left(\frac{s\left|B\left(x_{0}, r\right)\right|}{F(t)}\right)^{2 / \nu}:=m\left(s, t, x_{0}\right) .
$$

Plugging this into (2.7), we obtain

$$
I(t) \leq \frac{\int_{\{x \mid u(x, t)>s\}}|\nabla u(x, t)|^{2} d \mu(x)}{m\left(s, t, x_{0}\right)}+2 s F(t) .
$$

Hence

$$
\int_{\{x \mid u(x, t)>s\}}|\nabla u(x, t)|^{2} d \mu(x) \geq(I(t)-2 s F(t)) m\left(s, t, x_{0}\right) .
$$

The combination of (2.9) and (2.6) yields

$$
I^{\prime}(t) \leq-2(1-A)(I(t)-2 s F(t)) m\left(s, t, x_{0}\right) .
$$

Choosing $s$ so that $s F(t)=I(t) / 4$ yields

$$
I^{\prime}(t) \leq-(1-A) I(t) m\left(s, t, x_{0}\right),
$$


for all $t>0$ and the corresponding $s$.

We have that

$$
I(t)=p_{2 t}^{V}\left(x_{0}, x_{0}\right) \geq c / t^{\nu / 2}
$$

for $t \geq 1$. Indeed, since $V \geq 0$, by the maximum principle $p_{t}^{V}\left(x, x_{0}\right) \geq$ $p_{t}\left(x, x_{0}\right)$. Now it is well known (see [2]) that Assumptions (A) and (B) imply

$$
p_{2 t}\left(x_{0}, x_{0}\right) \geq \frac{c}{\left|B\left(x_{0}, \sqrt{t}\right)\right|} .
$$

One concludes by using Assumptions (A) and (D).

Let us now estimate $m\left(s, t, x_{0}\right)$. First, for $t \geq 1$,

$$
\begin{aligned}
c \sqrt{t} \leq r & =\sqrt{c_{2}^{-1} t\left(\left|\ln \left(C_{1} / s\right)\right|+1\right)} \\
& =\sqrt{c_{2}^{-1} t\left(\left|\ln \left(4 C_{1} F(t) / I(t)\right)\right|+1\right)} \\
& \leq \sqrt{c_{2}^{-1} t\left(\left|\ln \left(C F(t) t^{\nu / 2}\right)\right|+1\right)} \\
& \leq C \sqrt{t} \sqrt{\ln (e+t F(t))} .
\end{aligned}
$$

Finally,

$$
m\left(s, t, x_{0}\right) \geq \frac{c}{t \ln (e+t F(t))}\left(\frac{I(t)\left|B\left(x_{0}, \sqrt{t}\right)\right|}{F^{2}(t)}\right)^{2 / \nu} .
$$

By (2.10) and (2.11), it follows that

$$
I^{\prime}(t) \leq-c \frac{I(t)^{1+(2 / \nu)}\left|B\left(x_{0}, \sqrt{t}\right)\right|^{2 / \nu}}{t F(t)^{4 / \nu} \ln (e+t F(t))}
$$

that is

$$
\frac{I^{\prime}(t)}{I(t)^{1+(2 / \nu)}} \leq-\frac{c\left|B\left(x_{0}, \sqrt{t}\right)\right|^{2 / \nu}}{t F(t)^{4 / \nu} \ln (e+t F(t))} .
$$

Integrating (2.12) from $t / 2$ to $t$ and using the monotonicity of $F(t)$ and $\left|B\left(x_{0}, \sqrt{t}\right)\right|$, one easily obtains

$$
p_{2 t}^{V}\left(x_{0}, x_{0}\right)=I(t) \leq C \frac{F^{2}(t)[\ln (e+t F(t))]^{\nu / 2}}{\left|B\left(x_{0}, \sqrt{t}\right)\right|} .
$$

From this on-diagonal bound, under condition (2.4), one can derive the off-diagonal bound

$$
p_{t}^{V}(x, y) \leq C \frac{F^{2}(t)[\ln (e+t F(t))]^{\nu / 2}}{|B(x, \sqrt{t})|} \exp \left(-c d^{2}(x, y) / t\right),
$$

by either the method in [20] or the wave equation method in [34]. On the other hand, (2.4) implies $F(t) \leq C t^{N}$ for all $t \geq 1$ and some $N>0$; therefore the estimate takes the simpler form (2.5).

q.e.d. 


\subsection{The $L^{1}$ to $L^{1}$ estimates.}

Proposition 2.3. Suppose that $M$ satisfies Assumptions (A), (B), (C), (D), and that the operator $\Delta-V$ is strongly positive. If $V \in$ $L^{p}(M, \mu)$ for some $p \in[1,+\infty)$, then there exists $C=C(p)$ such that

$$
\begin{cases}\left\|P_{t}^{V}\right\|_{1,1} \leq C t^{1 / 2}, \forall t \geq 1, & \text { if } p=1, \\ \left\|P_{t}^{V}\right\|_{1,1} \leq C t^{(p-\theta) / 2}, \forall t \geq 1, & \text { if } 1<p<2, \text { for some } \theta=\theta(p)>0, \\ \left\|P_{t}^{V}\right\|_{1,1} \leq C t^{(p-1) / 2}, \forall t \geq 1, & \text { if } p \geq 2 .\end{cases}
$$

Remark. We shall see in Section 5 below that, if one does not assume (A) and (B), but only (C), (D) and strong positivity, one can still prove

$$
\left\|P_{t}^{V}\right\|_{1,1} \leq C_{p} t^{p / 2}, \forall t \geq 1,1 \leq p<+\infty .
$$

If now one only assumes that $\Delta-V$ is positive instead of being strongly positive, then one can still prove, for $V \in L^{p}(M, \mu)$ and $t \geq 1$,

$$
\left\|P_{t}^{V}\right\|_{1,1} \leq\left\{\begin{array}{l}
C t, \text { if } 1 \leq p \leq 2 \\
C t^{p / 2}, \text { if } p \geq 2
\end{array}\right.
$$

Note that, according to [35], Theorem 3.1, the above estimate is sharp in the range $1 \leq p \leq 2$, which shows the role of strong positivity in the better estimate of Proposition 2.3. For $p>2$, similar estimates were proved in [25], Theorem 8.1, by extending the method of [14], Theorem 3, to the manifold case. This is also what we shall do to prove the more precise Proposition 2.3, taking advantage in addition of the strong positivity of $V$.

Note added in proof: Under the assumption that $\Delta-V$ is positive (but without assuming strong positivity), the paper [28] contains better estimates than ours in the case when $p>\frac{\nu}{2}+1$, where $\nu$ is the doubling exponent in (1.2).

Proof. Let us first prove that there exists $\delta>0$ such that

$$
\sup _{y}\left\|p_{t}^{V}(., y)\right\|_{2}=\left\|P_{t}^{V}\right\|_{2, \infty} \leq C t^{-\delta}, \quad t \geq 1
$$

The proof goes as follows.

Given $f \in C_{0}^{\infty}(M)$ and $q>1$, by an easy consequence of the FeynmanKac formula (see for instance [41], p. 712), one may write

$$
\left|P_{t}^{V} f(x)\right| \leq\left[e^{-t(\Delta-q V)}|f|(x)\right]^{1 / q}\left[e^{t \Delta}|f|(x)\right]^{(q-1) / q} .
$$

Since $\Delta-V$ is strongly positive, when $q$ is sufficiently close to 1 , the operator $\Delta-q V$ is also strongly positive, thus $P_{t}^{q V}$ is contractive on $L^{2}(M, \mu)$. Hence

$$
\begin{aligned}
e^{-t(\Delta-q V)}|f|(x) \leq\left\|P_{t}^{q V}\right\|_{2, \infty}\|f\|_{2} & \leq\left\|P_{t-1}^{q V}\right\|_{2,2}\left\|P_{1}^{q V}\right\|_{2, \infty}\|f\|_{2} \\
& \leq\left\|P_{1}^{q V}\right\|_{2, \infty}\|f\|_{2},
\end{aligned}
$$


for $t \geq 1$. Using the assumption that $p_{t}$ is bounded from above by a Gaussian (Assumption (B)), we have

$$
e^{-t \Delta}|f|(x) \leq \sup _{y}\left\|p_{t}(y, .)\right\|_{2}\|f\|_{2} \leq \frac{C}{\sqrt{|B(x, \sqrt{t})|}}\|f\|_{2} .
$$

By Assumptions (A) and (D),

$$
|B(x, \sqrt{t})| \geq c_{1} t^{\nu / 2}|B(x, 1)| \geq c_{2} t^{\nu / 2} .
$$

Therefore,

$$
e^{t \Delta}|f|(x) \leq \frac{C}{t^{\nu / 4}}\|f\|_{2} .
$$

Substituting this and (2.16) into (2.15), we have, for some $\delta>0$,

$$
\left|P_{t}^{V} f(x)\right| \leq \frac{C}{t^{\delta}}\|f\|_{2}, \quad t \geq 1, x \in M
$$

This proves (2.14).

Now we are ready to prove the decay estimates in $L^{1}-L^{1}$ norm.

Case 1. Assume $V \in L^{1}(M, \mu)$.

Fix $y \in M$, and let $u(x, t)=p_{t}^{V}(x, y)$. Since $u$ satisfies

$$
\Delta u-V u+u_{t}=0
$$

integrating on $[1, t] \times M$ yields

$$
\int_{M} u(x, t) d \mu(x)=\int_{M} u(x, 1) d \mu(x)+\int_{1}^{t} \int_{M} V(x) u(x, s) d \mu(x) d s .
$$

By (2.1) and doubling, it follows that

$$
\int_{M} u(x, t) d \mu(x) \leq C+\int_{1}^{t} \int_{M} V(x) u(x, s) d \mu(x) d s .
$$

From (2.17) and the strong positivity of $V$,

$$
\begin{aligned}
& \int_{M} u(x, t) d \mu(x) \\
& \leq C+\int_{1}^{t} \int_{M} V(x) u(x, s) d \mu(x) d s \\
& \leq C+\left(\int_{1}^{t} \int_{M} V(x) d \mu(x) d s\right)^{1 / 2}\left(\int_{1}^{t} \int_{M} V(x) u^{2}(x, s) d \mu(x) d s\right)^{1 / 2} \\
& \leq C+\sqrt{A} \sqrt{t}\|V\|_{1}^{1 / 2}\left(\int_{1}^{t} \int_{M}|\nabla u|^{2}(x, s) d \mu(x) d s\right)^{1 / 2} .
\end{aligned}
$$

Multiplying by $u$ the equation

$$
\Delta u-V u+u_{t}=0
$$


and integrating on $[1, t] \times M$, we obtain

$$
\begin{aligned}
& \int_{1}^{t} \int_{M}|\nabla u|^{2}(x, s) d \mu(x) d s-\int_{1}^{t} \int_{M} V u^{2} d \mu(x) d s+\frac{1}{2} \int_{M} u^{2}(x, t) d \mu(x) \\
& =\frac{1}{2} \int_{M} u^{2}(x, 1) d \mu(x) .
\end{aligned}
$$

Since $\Delta-V$ is strongly positive, we obtain

$$
\int_{1}^{t} \int_{M}|\nabla u|^{2}(x, s) d \mu(x) d s \leq \frac{1}{2(1-A)} \int_{M} u^{2}(x, 1) d \mu(x) \leq \frac{C}{2(1-A)} .
$$

Finally

$$
\left\|P_{t}^{V}\right\|_{1 \rightarrow 1}=\int_{M} u(x, t) d \mu(x) \leq C \sqrt{\frac{A}{1-A}}\|V\|_{1}^{1 / 2} \sqrt{t},
$$

which is the claim.

Case 2. Now we assume that $V \in L^{p}(M, \mu), p \in(1,2)$.

The decay estimate in this range of $p$ seems to be new even in the Euclidean case.

Using Hölder's inequality repeatedly, one has

$$
\begin{aligned}
\int_{M} u(x, t) d \mu(x) \leq C+\int_{1}^{t} \int_{M} V(x) u(x, s) d \mu(x) d s \\
=C+\int_{1}^{t} \int_{M} V(x)^{p / 2} V(x)^{1-(p / 2)} u(x, s) d \mu(x) d s \\
\leq C+\left(\int_{1}^{t} \int_{M} V(x)^{p} d \mu(x) d s\right)^{1 / 2} \\
\quad \cdot\left(\int_{1}^{t} \int_{M} V(x)^{2-p} u^{2}(x, s) d \mu(x) d s\right)^{1 / 2} \\
=C+t^{1 / 2}\|V\|_{p}^{p / 2}\left(\int_{1}^{t} \int_{M} V(x)^{2-p} u(x, s)^{2(2-p)} u(x, s)^{2 p-2} d \mu(x) d s\right)^{1 / 2} \\
\leq C+t^{1 / 2}\|V\|_{p}^{p / 2}\left(\int_{1}^{t} \int_{M}\left[V(x)^{2-p} u(x, s)^{2(2-p)}\right]^{1 /(2-p)} d \mu(y) d s\right)^{(2-p) / 2} \\
\quad \cdot\left(\int_{1}^{t} \int_{M} u(x, s)^{(2 p-2) /(p-1)} d \mu(x) d s\right)^{(p-1) / 2} \\
=C+t^{1 / 2}\|V\|_{p}^{p / 2}\left(\int_{1}^{t} \int_{M} V(x) u^{2}(x, s) d \mu(y) d s\right)^{(2-p) / 2} \\
\quad \cdot\left(\int_{1}^{t} \int_{M} u^{2}(x, s) d \mu(x) d s\right)^{(p-1) / 2}
\end{aligned}
$$




$$
\begin{aligned}
\leq C & +A^{(2-p) / 2} t^{1 / 2}\|V\|_{p}^{p / 2}\left(\int_{1}^{t} \int_{M}|\nabla u(x, s)|^{2} d \mu(y) d s\right)^{(2-p) / 2} \\
& \cdot\left(\int_{1}^{t} \int_{M} u^{2}(x, s) d \mu(x) d s\right)^{(p-1) / 2} \cdot
\end{aligned}
$$

Using (2.18), we obtain

$$
\int_{M} u(x, t) d \mu(x) \leq C+C^{\prime} t^{1 / 2}\left(\int_{1}^{t} \int_{M} u^{2}(x, s) d \mu(x) d s\right)^{(p-1) / 2}
$$

From (2.14), we know that

$$
\int_{1}^{t} \int_{M} u^{2}(x, s) d \mu(x) d s \leq C t^{1-\delta},
$$

and using (2.18), we deduce

$$
\int_{M} u(x, t) d \mu(x) \leq C+C^{\prime} t^{1 / 2+(1-2 \delta)(p-1) / 2}=C t^{(p-\theta) / 2},
$$

with $\theta=2 \delta(p-1)$.

Case 3. The only remaining case is when $V \in L^{p}(M, \mu), p \geq 2$.

This case may be skipped in a first reading ; indeed, if one is prepared to replace $(p-1) / 2$ by $p / 2$ in the estimate, the simpler proof in Section 5 will do.

The reader may guess that the claimed estimate follows from the idea in [14], p. 99, where a similar bound for the Schrödinger heat kernel was proven in the Euclidean case. However, in [14], the authors use the boundedness of $\Delta^{-1 / 2}$ from some $L^{p}$ space to another. But it is known that this property is false for most open manifolds. Therefore, we have to work considerably harder. We will show that the inverse square root of the Laplacian on $M \times \mathbb{R}^{3}$ is bounded from the space $L^{p_{1}} \cap L^{p_{2}}$ to $L^{2}$ for some $p_{1}, p_{2}$. Then we will use the idea in [14] to get an $L^{1}$ to $L^{1}$ bound for a version of our Schrödinger semigroup acting on $N \equiv M \times \mathbb{R}^{3}$. After integrating over $\mathbb{R}^{3}$, we will reach the desired $L^{1}$ to $L^{1}$ bound for the Schrödinger semigroup acting on $M$.

Points in $N$ will be denoted by $\tilde{x}=\left(x, x^{\prime}\right)$ and $\tilde{y}=\left(y, y^{\prime}\right), \ldots$, where $x, y \in M$ and $x^{\prime}, y^{\prime} \in \mathbb{R}^{3}$. The distance function on $N$ is denoted by $d(\tilde{x}, \tilde{y})=d(x, y)+\left|x^{\prime}-y^{\prime}\right|$, and the Riemannian measure on $N$ by $d \tilde{\mu}$. The Laplace-Beltrami operator on $N$ will be denoted by $\tilde{\Delta}$. Denote by $\tilde{P}_{t}$ the heat semigroup on $N$ and let $\tilde{P}_{t}^{V}=e^{-t(\tilde{\Delta}-V)}$, where $V$ is the function on $M$ defined in (1.1). The kernel for $\tilde{\Delta}^{-1 / 2}$ is

$$
\Delta^{-1 / 2}(\tilde{x}, \tilde{y})=\int_{0}^{\infty} t^{-1 / 2} \tilde{p}_{t}(\tilde{x}, \tilde{y}) d t .
$$

It is clear that $N$ satisfies Assumptions (A), (B), (C) and (D) just like $M$ does. Moreover, if $\Delta-V$ is strongly positive on $M$, then $\tilde{\Delta}-V$ 
is strongly positive on $N$. Now, suppose we can prove that

$$
\left\|\tilde{P}_{t}^{V}\right\|_{1,1} \leq C t^{(p-1) / 2} \text {. }
$$

Then, since

$$
\tilde{p}_{t}^{V}(\tilde{x}, \tilde{y})=p_{t}^{V}(x, y) \frac{c}{t^{3 / 2}} e^{-\left|x^{\prime}-y^{\prime}\right|^{2} / 4 t},
$$

we will deduce that

$$
\begin{aligned}
\left\|P_{t}^{V}\right\|_{1,1}=\left\|P_{t}^{V}\right\|_{\infty, \infty} & =\left\|P_{t}^{V} 1\right\|_{\infty}=\left\|\tilde{P}_{t}^{V} 1\right\|_{\infty} \\
& =\left\|\tilde{P}_{t}^{V}\right\|_{\infty, \infty}=\left\|\tilde{P}_{t}^{V}\right\|_{1,1} \leq C t^{(p-1) / 2},
\end{aligned}
$$

thus finishing Case 3. The rest of the section is devoted to proving (2.20). It will be divided into several steps.

Step 1 . We show that there exists $C>0$ such that

$$
\tilde{\Delta}^{-1 / 2}(\tilde{x}, \tilde{y}) \leq C \frac{d(\tilde{x}, \tilde{y})}{|B(\tilde{x}, d(\tilde{x}, \tilde{y}))|} .
$$

From the upper bound for $\tilde{P}_{t}$, which is a consequence of the Gaussian upper bound for $P_{t}$, we have

$$
\begin{aligned}
I & \equiv \tilde{\Delta}^{-1 / 2}(\tilde{x}, \tilde{y}) \\
& \leq C \int_{0}^{\infty} \frac{1}{\sqrt{t}} \frac{e^{-c d^{2}(\tilde{x}, \tilde{y}) / t}}{|B(\tilde{x}, \sqrt{t})|} d t \\
& =C \int_{0}^{d^{2}(\tilde{x}, \tilde{y})} \frac{1}{\sqrt{t}} \frac{e^{-c d^{2}(\tilde{x}, \tilde{y}) / t}}{|B(\tilde{x}, \sqrt{t})|} d t+C \int_{d^{2}(\tilde{x}, \tilde{y})}^{\infty} \frac{1}{\sqrt{t}} \frac{e^{-c d^{2}(\tilde{x}, \tilde{y}) / t}}{|B(\tilde{x}, \sqrt{t})|} d t \\
& \equiv C I_{1}+C I_{2} .
\end{aligned}
$$

By the doubling condition, for $t \leq d^{2}(\tilde{x}, \tilde{y})$,

$$
|B(\tilde{x}, \sqrt{t})| \geq c|B(\tilde{x}, d(\tilde{x}, \tilde{y}))|\left(\frac{\sqrt{t}}{d(\tilde{x}, \tilde{y})}\right)^{\nu} .
$$

Hence,

$$
I_{1} \leq C \frac{d(\tilde{x}, \tilde{y})}{|B(\tilde{x}, d(\tilde{x}, \tilde{y}))|}
$$

Next we estimate $I_{2}$.

By (2.21), we have

$$
\begin{aligned}
I_{2} \leq C \int_{d^{2}(\tilde{x}, \tilde{y})}^{\infty} \frac{1}{\sqrt{t}} \frac{1}{|B(x, \sqrt{t})| t^{3 / 2}} d t & \leq \frac{C}{|B(x, d(\tilde{x}, \tilde{y}))|} \int_{d^{2}(\tilde{x}, \tilde{y})}^{\infty} \frac{d t}{t^{2}} \\
& =\frac{C}{|B(x, d(\tilde{x}, \tilde{y}))| d^{2}(\tilde{x}, \tilde{y})} .
\end{aligned}
$$

Note that

$$
|B(\tilde{x}, d(\tilde{x}, \tilde{y}))|=|B(x, d(\tilde{x}, \tilde{y}))| d^{3}(\tilde{x}, \tilde{y}) .
$$


The above implies

$$
I_{2} \leq \frac{C d(\tilde{x}, \tilde{y})}{|B(\tilde{x}, d(\tilde{x}, \tilde{y}))|}
$$

The combination of (2.24) and (2.23) implies (2.22).

Step 2 . We prove that there exist $p_{1}, p_{2}>1$ and $C>0$ such that

$$
\left\|\tilde{\Delta}^{-1 / 2} f\right\|_{2} \leq C\left(\|f\|_{p_{1}}+\|f\|_{p_{2}}\right),
$$

for all $f \in L^{p_{1}} \cap L^{p_{2}}$.

From (2.22),

$$
\begin{aligned}
\left|\tilde{\Delta}^{-1 / 2} f(\tilde{x})\right| & \leq C \int_{\tilde{M}} \frac{d(\tilde{x}, \tilde{y})}{|B(\tilde{x}, d(\tilde{x}, \tilde{y}))|}|f(\tilde{y})| d \tilde{\mu}(\tilde{y}) \\
& \leq C \int_{d(\tilde{x}, \tilde{y}) \leq 1} \cdots+C \int_{d(\tilde{x}, \tilde{y}) \geq 1} \equiv C J_{1}(\tilde{x})+C J_{2}(\tilde{x}) .
\end{aligned}
$$

By Young's inequality,

$$
\left\|J_{1}\right\|_{2} \leq C\|f\|_{p_{1}} \sup _{\tilde{x}}\left(\int_{d(\tilde{x}, \tilde{y}) \leq 1}\left[\frac{d(\tilde{x}, \tilde{y})}{|B(\tilde{x}, d(\tilde{x}, \tilde{y}))|}\right]^{r_{1}} d \tilde{\mu}(\tilde{y})\right)^{1 / r_{1}}
$$

where $\left(1 / p_{1}\right)+\left(1 / r_{1}\right)=1+(1 / 2)$. Hence,

$$
\begin{aligned}
\left\|J_{1}\right\|_{2} & \leq\|f\|_{p_{1}} \sup _{\tilde{x}} \sum_{k=0}^{\infty}\left(\int_{2^{-(k+1)} \leq d(\tilde{x}, \tilde{y}) \leq 2^{-k}}\left[\frac{d(\tilde{x}, \tilde{y})}{|B(\tilde{x}, d(\tilde{x}, \tilde{y}))|}\right]^{r_{1}} d \tilde{\mu}(\tilde{y})\right)^{1 / r_{1}} \\
& \leq C\|f\|_{p_{1}} \sup _{\tilde{x}} \sum_{k=0}^{\infty} \frac{2^{-k r_{1}}}{\left|B\left(\tilde{x}, 2^{-(k+1)}\right)\right|^{r_{1}-1}} .
\end{aligned}
$$

Using the doubling property and the fact that, thanks to Assumption (D), $|B(\tilde{x}, 1)| \geq c>0$, we have

$$
\left|B\left(\tilde{x}, 2^{-(k+1)}\right)\right| \geq C 2^{-k \nu}|B(\tilde{x}, 1)| \geq C 2^{-k \nu} .
$$

Hence,

$$
\left\|J_{1}\right\|_{2} \leq\|f\|_{p_{1}} \sum_{k=0}^{\infty} 2^{-k r_{1}} 2^{k \mu\left(r_{1}-1\right)}
$$

Choosing $r_{1}$ sufficiently close to 1 , the above series is convergent. Therefore

$$
\left\|J_{1}\right\|_{2} \leq C\|f\|_{p_{1}} .
$$

Similarly, by Young's inequality again,

$$
\left\|J_{2}\right\|_{2} \leq C\|f\|_{p_{2}} \sup _{\tilde{x}}\left(\int_{d(\tilde{x}, \tilde{y}) \geq 1}\left[\frac{d(\tilde{x}, \tilde{y})}{|B(\tilde{x}, d(\tilde{x}, \tilde{y}))|}\right]^{r_{2}} d \tilde{\mu}(\tilde{y})\right)^{1 / r_{2}}
$$


where $\left(1 / p_{2}\right)+\left(1 / r_{2}\right)=1+(1 / 2)$. Hence,

$$
\begin{aligned}
\left\|J_{2}\right\|_{2} & \leq C\|f\|_{p_{2}} \sup _{\tilde{x}} \sum_{k=0}^{\infty}\left(\int_{2^{k} \leq d(\tilde{x}, \tilde{y}) \leq 2^{k+1}}\left[\frac{d(\tilde{x}, \tilde{y})}{|B(\tilde{x}, d(\tilde{x}, \tilde{y}))|}\right]^{r_{2}} d \tilde{\mu}(\tilde{y})\right)^{1 / r_{2}} \\
& \leq C\|f\|_{p_{2}} \sup _{\tilde{x}} \sum_{k=0}^{\infty} \frac{2^{k r_{2}}}{\left|B\left(\tilde{x}, 2^{k}\right)\right|^{r_{2}-1}} .
\end{aligned}
$$

Using the fact that $\left|B\left(\tilde{x}, 2^{k}\right)\right| \geq c 2^{3 k}$, which follows from the definition of $N$ and Assumption (D), we have

$$
\left\|J_{2}\right\|_{2} \leq\|f\|_{p_{2}} \sum_{k=0}^{\infty} 2^{k r_{2}-3 k\left(r_{2}-1\right)} .
$$

Choosing $r_{2}$ sufficiently large, the above series is convergent. Therefore

$$
\left\|J_{2}\right\|_{2} \leq C\|f\|_{p_{2}} .
$$

Inequality (2.25) immediately follows from (2.27) and (2.26).

Step 3. As in [14], by Duhamel's formula, one has

$$
\begin{aligned}
\left\|\tilde{P}_{t+1}^{V}\right\|_{\infty, \infty} & =\left\|\tilde{P}_{t+1}^{V} 1\right\|_{\infty} \\
& \leq\left\|\tilde{P}_{1}^{V} 1\right\|_{\infty}+\int_{1}^{t}\left\|\tilde{P}_{s+1}^{V} V\right\|_{\infty} d s \\
& \leq C+\int_{1}^{t}\left\|\tilde{P}_{s+1}^{V} V\right\|_{\infty} d s ;
\end{aligned}
$$

hence by interpolation,

$$
\left\|\tilde{P}_{t+1}^{V}\right\|_{\infty, \infty} \leq C+\int_{1}^{t}\left\|\tilde{P}_{s+1}^{V}\right\|_{2, \infty}^{2 / p}\left\|\tilde{P}_{s+1}^{V}\right\|_{\infty, \infty}^{1-(2 / p)}\|V\|_{p} d s
$$

Let us now estimate $\left\|\tilde{P}_{s+1}^{V}\right\|_{2, \infty}^{2 / p}$. Using the strong positivity of $\tilde{\Delta}-V$ on $N$, one has

$$
(\tilde{\Delta}-V) \geq a^{2} \tilde{\Delta}
$$

for some $a>0$. Therefore, for all $f \in C_{0}^{\infty}(N)$,

$$
\left\|(\tilde{\Delta}-V)^{-1 / 2} f\right\|_{2} \leq a^{-1}\left\|\tilde{\Delta}^{-1 / 2} f\right\|_{2} \leq C\|f\|_{X} .
$$

Here $X=L^{p_{1}} \cap L^{p_{2}}$ where $p_{1}, p_{2}$ are given in (2.25) and

$$
\|f\|_{X}=\|f\|_{p_{1}}+\|f\|_{p_{2}} .
$$

Hence

$$
\begin{aligned}
\left\|e^{-t(\tilde{\Delta}-V)} f\right\|_{2} & =t^{-1 / 2}\left\|e^{-(\tilde{\Delta}-V) t}((\tilde{\Delta}-V) t)^{1 / 2}(\tilde{\Delta}-V)^{-1 / 2} f\right\|_{2} \\
& \leq C t^{-1 / 2}\left\|(\tilde{\Delta}-V)^{-1 / 2} f\right\|_{2} \leq\left\|\tilde{\Delta}^{-1 / 2} f\right\|_{2} \\
& \leq C t^{-1 / 2}\|f\|_{X}
\end{aligned}
$$

This shows

$$
\left\|\tilde{P}_{t}\right\|_{2, X^{*}}=\left\|\tilde{P}_{t}\right\|_{X, 2} \leq C t^{-1 / 2},
$$


where $X^{*}$ is the dual of $X$. Then write

$$
\left\|\tilde{P}_{s+1}^{V}\right\|_{2, \infty} \leq\left\|\tilde{P}_{s}^{V}\right\|_{2, X^{*}}\left\|\tilde{P}_{1}^{V}\right\|_{X^{*}, \infty} .
$$

It follows easily from the bound

$$
\tilde{p}_{1}^{V}(\tilde{x}, \tilde{y}) \leq \frac{C}{|B(\tilde{y}, 1)|} e^{-c d^{2}(\tilde{x}, \tilde{y})},
$$

and Assumptions (A) and (D) that $\tilde{P}_{1}^{V}$ is bounded from $L^{1}$ to any $L^{p}$, $1 \leq p \leq+\infty$. Therefore

$$
\left\|\tilde{P}_{1}^{V}\right\|_{X^{*}, \infty}=\left\|\tilde{P}_{1}^{V}\right\|_{1, X}=\left\|\tilde{P}_{1}^{V}\right\|_{1, p_{1}}+\left\|\tilde{P}_{1}^{V}\right\|_{1, p_{2}}<+\infty .
$$

This, together with (2.30), implies

$$
\left\|\tilde{P}_{s+1}^{V}\right\|_{2, \infty} \leq C s^{-1 / 2} .
$$

Using this and (2.28), we obtain

$$
\left\|\tilde{P}_{t+1}^{V}\right\|_{\infty, \infty} \leq C+C\|V\|_{p} \int_{1}^{t} s^{-1 / p}\left\|\tilde{P}_{s+1}^{V}\right\|_{\infty, \infty}^{1-(2 / p)} d s .
$$

By Gronwall's lemma,

$$
\left\|\tilde{P}_{t+1}^{V}\right\|_{\infty, \infty} \leq C t^{p / 2-1 / 2} .
$$

Since

$$
\left\|\tilde{P}_{t+1}^{V}\right\|_{1,1}=\left\|\tilde{P}_{t+1}^{V}\right\|_{\infty, \infty},
$$

the proof of Proposition 2.3 is complete.

q.e.d.

2.3. Proof of Theorem 2.2. Under the assumptions of Theorem 2.2, the combination of Propositions 2.2 and 2.3 yields

$$
p_{t}^{V}(x, y) \leq \frac{C t \ln ^{\nu / 2}(e+t)}{|B(x, \sqrt{t})|} \exp \left(-c d^{2}(x, y) / t\right), \forall x, y \in M, t \geq 1
$$

if $p=1$,

$$
p_{t}^{V}(x, y) \leq \frac{C t^{p-\theta}}{|B(x, \sqrt{t})|} \exp \left(-c d^{2}(x, y) / t\right), \forall x, y \in M, t \geq 1
$$

for some $\theta=\theta(p)>0$, if $1 \leq p<2$, and

$$
p_{t}^{V}(x, y) \leq \frac{C t^{p-1} \ln ^{\nu / 2}(e+t)}{|B(x, \sqrt{t})|} \exp \left(-c d^{2}(x, y) / t\right), \forall x, y \in M, t \geq 1
$$

if $p \geq 2$.

The above bound does not explicitly reflect the contribution of the constant $A$ (which measures the size of $V$ ). To remedy this, we again use (2.15), which yields

$$
p_{t}^{V}(x, y) \leq\left(p_{t}^{V^{\prime}}(x, y)\right)^{1 / q}\left(p_{t}(x, y)\right)^{(q-1) / q}, \forall x, y \in M, t>0,
$$

where $V^{\prime}=q V$ and $q>1$. 
Assume $A>0$, otherwise there is nothing to prove. If $q<1 / A$, then $V^{\prime}$ is obviously strongly positive with constant $q A<1$.

If $1<p<2,(2.33)$ yields

$$
p_{t}^{V^{\prime}}(x, y) \leq \frac{C t^{p-\theta}}{|B(x, \sqrt{t})|} \exp \left(-c d^{2}(x, y) / t\right)
$$

Applying (2.36) and using the Gaussian upper bound on $p_{t}$, we obtain

$$
p_{t}^{V}(x, y) \leq \frac{C t^{(p-\theta) / q}}{|B(x, \sqrt{t})|} \exp \left(-c d^{2}(x, y) / t\right) .
$$

Taking $q$ sufficiently close to $1 / A$, one can make $(p-\theta) / q<p A$, which finishes the proof of Theorem 2.2 in the case $1<p<2$. The proofs when $p=1$ and $p \geq 2$ are identical using (2.32) and (2.34) instead of $(2.33)$.

q.e.d.

\section{Gaussian bound on the heat kernel on forms and the Riesz transform}

Our next theorem provides an all time Gaussian upper bound for the heat kernel on 1-forms on complete non-compact Riemannian manifolds satisfying Assumptions (A), (B), (C), together with a certain condition of smallness of the Ricci curvature. Using this theorem and an argument in [11], one deduces a proper bound for the gradient of the heat kernel on functions. By the main result in [1], one obtains the $L^{p}$ boundedness of the Riesz transform on these manifolds for all $1<p<+\infty$. Let us point out that in [25], Theorem 9.1, another sufficient condition in terms of Ricci curvature is given for $L^{p}$ boundedness of the Riesz transform. However, this condition seems to exclude Ricci curvature bounded from below together with non-compactness.

Theorem 3.1. Let $M$ be a complete non-compact Riemannian manifold satisfying Assumptions (A), (B) and (C). Then there exists $\delta>0$ depending only on the constants in $(\mathrm{A})$ and $(\mathrm{B})$ such that for any $c \in$ $(0,1 / 4)$, there exists $C>0$ such that

$$
\left|\vec{p}_{t}(x, y)\right| \leq \frac{C}{|B(x, \sqrt{t})|} \exp \left(-c d(x, y)^{2} / t\right),
$$

for all $x, y \in M$ and $t>0$, provided that

$$
K(V) \equiv \sup _{x \in M} \int_{0}^{\infty} \int_{M} \frac{1}{|B(x, \sqrt{s})|} e^{-d^{2}(x, y) / s} V(y) d \mu(y) d s<\delta .
$$

In the Euclidean case and for certain potentials decaying sufficiently fast near infinity, the strong positivity of $\Delta-V$ is actually equivalent to the claim that $p_{t}^{V}$ has global Gaussian upper and lower bound. This fact was proven in [44], Theorem B. It can also be extended to the non-compact manifold case under suitable assumptions. For a recent 
development, see $[\mathbf{3 9}]$. These results clearly yield Gaussian upper bound for $\vec{p}_{t}$ by semigroup domination.

As we already said, the following statement is a consequence from Theorem 3.1 and either Theorem 5.5 in [11], or Theorem 1.4 in [1] together with [11], pp. 1740-1741. This extends the class of manifolds for which one can answer a question asked by Strichartz in [37].

Corollary 3.1. Let $M$ be a complete non-compact Riemannian manifold satisfying Assumptions (A), (B), (C), and condition (3.1) for $\delta>0$ small enough. Then, for all $p \in(0,+\infty)$, there exist $C_{p}, c_{p}>0$ such that

$$
c_{p}\||\nabla f|\|_{p} \leq\left\|\Delta^{1 / 2} f\right\|_{p} \leq C_{p}\||\nabla f|\|_{p}, \quad \forall f \in C_{0}^{\infty}(M) .
$$

Proof of Theorem 3.1. As we have seen in the introduction,

$$
\left|\vec{p}_{t}(x, y)\right| \leq p_{t}^{V}(x, y)
$$

under Assumptions (A) to (C). Now let us recall Theorem A, part (b) in $[\mathbf{4 0}]$. It implies that

$$
p_{t}^{V}(x, y) \leq \frac{C}{|B(x, \sqrt{t})|} e^{-c d^{2}(x, y) / t},
$$

for all $x, y \in M$ and $t>0$, provided that, for certain $c_{0}, \varepsilon_{0}>0$, there holds

$$
N(V)<\varepsilon_{0}
$$

Here,

$$
\begin{aligned}
N(V) \equiv \sup _{x \in M, t>0} \int_{0}^{t} \int_{M} \frac{e^{-c_{0} d^{2}(x, y) /(t-s)}}{|B(x, \sqrt{t-s})|} V(y) d \mu(y) d s \\
\quad+\sup _{y \in M, s>0} \int_{s}^{\infty} \int_{M} \frac{e^{-c_{0} d^{2}(x, y) /(t-s)}}{|B(x, \sqrt{t-s})|} V(x) d \mu(x) d t .
\end{aligned}
$$

We should mention that this theorem was stated for a doubling metric in the Euclidean space and for time dependent functions $V$, under the extra assumption $(D)$. However, the proof was a general one applicable verbatim to any manifold under Assumptions (A) and (B) only.

Changing variables and using doubling, one sees that

$$
N(V) \leq C_{0} K(V)
$$

where $C_{0}$ only depends on the doubling constants. The conclusion follows.

q.e.d.

\section{Remarks.}

- Suppose in addition that $|B(x, r)| \sim r^{n}$ with $n>2$, uniformly in $x \in M$, for large $r$; then it is any easy exercise to check that the theorem holds if

$$
V(x) \leq \frac{a}{1+d\left(x, x_{0}\right)^{2+b}}
$$


with any $b>0$ and $a$ sufficiently small, for some fixed $x_{0} \in M$.

- Let $\left(M, g_{0}\right)$ be a nonparabolic manifold with nonnegative Ricci curvature and volume growth property as in the last remark. Let $h$ be another metric and $\eta$ be a smooth cut-off function on $M$. Then the manifold $(M, g)$ with $g=g_{0}+\lambda \eta h$ is covered by the theorem when $\lambda \geq 0$ is sufficiently small. This is so because the constants in $(A)$ and $(B)$ are uniformly bounded when $0 \leq \lambda \leq 1$ while $V=V(x)$ (for the metric $g$ ), being a compactly supported function is arbitrarily small when $\lambda \rightarrow 0$.

\section{The case of non-negative Ricci curvature outside a compact set}

In the next theorem, we establish an upper bound for the heat kernel on 1-forms assuming Ricci curvature is nonnegative outside a compact set. The upshot of the theorem is that no other restriction on the Ricci curvature is needed. This upper bound gives a good control of the heat kernel even in the presence of harmonic forms. In general, one cannot expect the heat kernel on forms to decay to zero, due to the possible presence of $L^{2}$ harmonic forms. Here we are able to show that the heat kernel has certain spatial decay anyway. Using the spectral decomposition of heat kernels, one can see that the upper bound in the theorem below is quite sharp near the diagonal at least. As far as we know, this bound is new even for Schrödinger heat kernels in the Euclidean case.

Moreover, the assumption that the Ricci curvature is 0 outside of a compact set can be improved to assuming that the negative part of the Ricci curvature decays sufficiently fast near infinity. But we will not seek the full generality this time.

Theorem 4.1. Let $M$ be a manifold satisfying Assumptions (A), (B) and (D). In addition, we assume that the Ricci curvature of $M$ is nonnegative outside a compact set and the manifold is nonparabolic. Then, for a fixed $0 \in M$, there exist $C, c>0$ such that

$$
\left|\vec{p}_{t}(x, y)\right| \leq C \min \{\Gamma(x, 0) \Gamma(y, 0), 1\} e^{-c d^{2}(x, y) / t}+\frac{C}{|B(x, \sqrt{t})|} e^{-c d^{2}(x, y) / t}
$$

for all $x, y \in M, t>1$. Here $\Gamma$ is the Green's function of the Laplacian $\Delta$ on $M$.

\section{Proof.}

We divide the proof into two steps.

Step 1.

As in Section 2.1, we need a preliminary estimate.

$$
\left|\vec{p}_{t}(x, y)\right| \leq C e^{-c d^{2}(x, y) / t}, t \geq 1 \text {, }
$$


for some $C, c>0$. Note that this estimate does not follow from Proposition 2.1 and (1.3), since we do not assume strong positivity of $\Delta-V$ any more.

However, the method is very similar to the one in Proposition 2.1. Let $u_{0}$ be a smooth compactly supported 1 -form. Write

$$
u(x, t)=\vec{P}_{t} u_{0}(x) .
$$

Direct computations show, for any fixed $y \in M$ and $D>0$,

$$
\begin{aligned}
& \frac{d}{d t} \int_{M}|u|^{2} e^{\frac{d^{2}(x, y)}{D t}} d \mu(x) \\
& =-2 \int_{M} e^{\frac{d^{2}(x, y)}{D t}} u \cdot \vec{\Delta} u d \mu(x)-\int_{M}|u|^{2} e^{\frac{d^{2}(x, y)}{D t}} \frac{d^{2}(x, y)}{D t^{2}} d \mu(x) .
\end{aligned}
$$

Noticing that $\vec{\Delta}=d^{*} d+d d^{*}$, the above implies, after integration by parts,

$$
\begin{aligned}
\frac{d}{d t} \int_{M}|u|^{2} e^{\frac{d^{2}(x, y)}{D t}} d \mu(x) \\
=-2 \int_{M} e^{\frac{d^{2}(x, y)}{D t}}\left(d\left(\frac{d^{2}(x, y)}{D t}\right) \wedge u\right) \cdot d u d \mu(x) \\
\quad-2 \int_{M} e^{\frac{d^{2}(x, y)}{D t}} d u \cdot d u d \mu(x) \\
\quad-2 \int_{M} e^{\frac{d^{2}(x, y)}{D t}}\left|d^{*} u\right|^{2} d \mu(x)-\int_{M}|u|^{2} e^{\frac{d^{2}(x, y)}{D t}} \frac{d^{2}(x, y)}{D t^{2}} d \mu(x) \\
\leq C \int_{M} e^{\frac{d^{2}(x, y)}{D t}} \frac{d(x, y)}{D t}|u||d u| d \mu(x)-2 \int_{M} e^{\frac{d^{2}(x, y)}{D t}}|d u|^{2} d \mu(x) \\
\quad-\int_{M}|u|^{2} e^{\frac{d^{2}(x, y)}{D t}} \frac{d^{2}(x, y)}{D t^{2}} d \mu(x) .
\end{aligned}
$$

Using the inequality $\frac{d(x, y)}{D t}|u||d u| \leq \frac{1}{\varepsilon} \frac{d^{2}(x, y)}{D^{2} t^{2}}|u|^{2}+\varepsilon|d u|^{2}$, we find that

$$
\frac{d}{d t} \int_{M}|u|^{2} e^{\frac{d^{2}(x, y)}{D t}} d \mu(x) \leq 0
$$

when $D$ is sufficiently large.

Letting $u_{0}$ range over a suitable family of forms concentrated around $y$, we obtain

$$
\int_{M}\left|\vec{p}_{t}(x, y)\right|^{2} e^{\frac{d^{2}(x, y)}{D t}} d \mu(x) \leq \int_{M}\left|\vec{p}_{1}(x, y)\right|^{2} e^{\frac{d^{2}(x, y)}{D}} d \mu(x)
$$

when $t \geq 1$. By the semigroup domination property (1.3), Assumptions (C) and (B),

$$
\left|\vec{p}_{1}(x, y)\right| \leq p_{1}^{V}(x, y) \leq C p_{1}(x, y) \leq \frac{C}{|B(x, 1)|} e^{-c d^{2}(x, y)} .
$$


Integrating and using Assumptions (D) and (A), we have, for a suitable $D>0$,

$$
\int_{M}\left|\vec{p}_{t}(x, y)\right|^{2} e^{\frac{d^{2}(x, y)}{D t}} d \mu(x) \leq C, \quad t \geq 1 .
$$

Next, using the semigroup property

$$
\begin{aligned}
& \left|\vec{p}_{2 t}(x, y)\right| \\
& =\left|\int_{M} \vec{p}_{t}(x, z) \vec{p}_{t}(z, y) d \mu(z)\right| \\
& =\left|\int_{M} e^{\frac{d^{2}(x, z)}{2 D t}} \vec{p}_{t}(x, z) e^{\frac{d^{2}(z, y)}{2 D t}} \vec{p}_{t}(z, y) e^{-\frac{d^{2}(x, z)}{2 D t}-\frac{d^{2}(z, y)}{2 D t}} d \mu(z)\right| \\
& \leq e^{-\frac{d^{2}(x, y)}{4 D t}}\left[\int_{M} e^{\frac{d^{2}(x, z)}{D t}}\left|\vec{p}_{t}(x, z)\right|^{2} d \mu(z)\right]^{1 / 2} \\
& \quad \cdot\left[\int_{M} e^{\frac{d^{2}(y, z)}{D t}}\left|\vec{p}_{t}(y, z)\right|^{2} d \mu(z)\right]^{1 / 2} .
\end{aligned}
$$

Together with (4.2), this implies (4.1).

Step 2.

We assume that the Ricci curvature is nonnegative outside of a ball $B(0, A)$ for a fixed $A>0$. Write, for any given $x_{0} \in \partial B(0, A)$,

$$
u(y, t)=\left|\vec{p}_{t}\left(x_{0}, y\right)\right| \text {. }
$$

Then it is an immediate consequence of Bochner's formula (see for instance [15], Lemma 4.1) that $u$ is a subsolution of the scalar heat equation in $B^{c}(0, A) \times(0, \infty)$, i.e.,

$$
\Delta u(y, t)+u_{t}(y, t) \leq 0 .
$$

Since, according to (4.1), $u$ is bounded from above by a constant for $t \geq 1$, and $\Gamma(y, 0)$ is bounded from below by a positive constant on any compact set, there exists $C>0$ such that

$$
u(y, t) \leq C \Gamma(y, 0), \forall y \in \partial B(0, A), t \geq 1 .
$$

Moreover, using again (4.1),

$$
u(y, 1) \leq C e^{-c d^{2}\left(y, x_{0}\right)} \leq C^{\prime} \Gamma(y, 0)
$$

for $y \in B^{c}(0, A)$. The last inequality is due to the Cheng-Yau gradient estimate from [7], which implies

$$
\Gamma(y, 0) \geq c e^{-C d(y, 0)}
$$

for some positive constants $C, c>0$ and $y \in B^{c}(0, A)$. Now by the maximum principle, using (4.1) again, we deduce

$$
u(y, t) \leq C \Gamma(y, 0)
$$


for all $t \geq 1$ and $y \in B^{c}(0, A)$. Here we just used the simple observation that $\Gamma(y, 0)$ is a solution of the scalar heat equation, whereas $u$ is a subsolution as already observed.

We have proved that

$$
\left|\vec{p}_{t}\left(x_{0}, y\right)\right| \leq C \Gamma(y, 0)
$$

for all $t \geq 1, y \in B^{c}(0, A)$ and $x_{0} \in \partial B(0, A)$. Let us now explain how to keep the same estimate while moving away $x_{0}$.

For a fixed $y \in B^{c}(0, A)$, define the function

$$
w(x, t)=\left|\vec{p}_{t}(x, y)\right| \text {. }
$$

Then $w$ is a subsolution of the scalar heat equation on $B^{c}(0, A) \times$ $(0,+\infty)$. For $x \in \partial B(0, A)$, by the above estimate on $u$ we have

$$
w(x, t)=\left|\vec{p}_{t}(x, y)\right| \leq C \Gamma(y, 0) .
$$

Since $\Gamma(x, 0)$ is bounded away from 0 for $x \in \partial B(0, A)$, it holds

$$
w(x, t) \leq C^{\prime} \Gamma(y, 0) \Gamma(x, 0)
$$

for some $C^{\prime}>0$. It is clear that the function

$$
h(x, t)=\int_{M} p_{t-1}(x, z) w(z, 1) d \mu(z)+C \Gamma(y, 0) \Gamma(x, 0)
$$

is a solution of the scalar heat equation in $B^{c}(0, A) \times[1, \infty)$. Moreover, on the parabolic boundary of the region, $h$ dominates $w$. By the maximum principle again

$$
w(x, t) \leq h(x, t)=\int_{M} p_{t-1}(x, z) w(z, 1) d \mu(z)+C \Gamma(y, 0) \Gamma(x, 0)
$$

for $x \in B^{c}(0, A)$ and $t \geq 1$. Next we estimate the above integral term in the following way, by using the Gaussian upper bound for $p_{t}$ :

$$
\begin{aligned}
& \int_{M} p_{t-1}(x, z) w(z, 1) d \mu(z) \\
& \leq \int p_{t-1}(x, z) e^{-c d(y, z)^{2}} d \mu(z) \\
& \leq \int_{d(x, z) \geq d(x, y) / 2} \cdots d \mu(z)+\int_{d(y, z) \geq d(x, y) / 2} \cdots d \mu(z) \\
& \leq \frac{C}{|B(x, \sqrt{t})|} .
\end{aligned}
$$

Finally, incorporating (4.1),

$$
\left|\vec{p}_{t}(x, y)\right|=w(x, t) \leq C \min \{\Gamma(x, 0) \Gamma(y, 0), 1\}+\frac{C}{|B(x, \sqrt{t})|}
$$

for all $x, y$ in $M$ and $t \geq 2$. This is the desired on-diagonal estimate.

Now the theorem follows from the standard process of going from on to off-diagonal estimate. See [34].

q.e.d. 


\section{Bounds on manifolds without doubling condition}

In this final section we turn to noncompact manifolds not necessarily satisfying the volume doubling condition. This class of manifolds offers a much richer variety than the doubling ones.

We show that under reasonable conditions the on-diagonal upper bound on the heat kernel on forms differs from that on functions only by a suitable power of time $t$.

Let us consider a $n$-dimensional manifold $M$ with Ricci curvature bounded from below, and whose small balls do not collapse, in other words Assumptions (C) and (D) are satisfied. Then

$$
p_{t}(x, y) \leq C t^{-n / 2} \exp \left(-c d^{2}(x, y) / t\right), \forall 0<t \leq 1, x, y \in M,
$$

for some $C, c>0$ and $p_{t}^{V}(x, y),\left|\vec{p}_{t}(x, y)\right|$ satisfy similar estimates.

Let us assume that the heat kernel on functions has a uniform rate of decay $\gamma$, where $\gamma$ is increasing, $C^{1}$ and one-to-one on $\mathbb{R}_{+}$:

$$
\sup _{x \in M} p_{t}(x, x) \leq \frac{1}{\gamma(t)}, \forall t>0 .
$$

According to [18], this implies the following so-called uniform FaberKrahn inequality: for any set $\Omega \subset M$,

$$
\lambda_{1}(\Omega) \geq \Lambda(|\Omega|),
$$

where $\Lambda$ is given by

$$
\Lambda(t)=\frac{\gamma^{\prime}(t)}{\gamma(t)}
$$

i.e.,

$$
t=\int_{0}^{\gamma(t)} \frac{d \eta}{\eta \Lambda(\eta)}
$$

Conversely, if $\gamma$ satisfies a mild condition, the converse is true. For more on this, as well as examples where one can compute $\Lambda$, therefore $\gamma$, see for instance $[\mathbf{9}]$.

We can now state our result in this setting.

Theorem 5.1. Suppose $M$ satisfies Assumptions (C) and (D). Assume that $V \in L^{p}(M, \mu)$ for some $p \in[1,+\infty)$, and that $\Delta-V$ is strongly positive. Finally assume that the heat kernel on functions on $M$ satisfies the estimate (5.2). Then there exist positive constants $c$ and $C$ such that

$$
\left|\vec{p}_{t}(x, y)\right| \leq \frac{C t^{p}}{\gamma(c t)}, \forall t \geq 1, x, y \in M .
$$

Proof. We divide the proof into two parts.

Step 1. $L^{1}$ to $L^{1}$ bound. 
Under the assumptions of the theorem we will prove that

$$
\left\|P_{t}^{V}\right\|_{1,1} \leq C t^{p / 2}, \forall t \geq 1 \text {, if } p \geq 1 .
$$

Comparing with the proof of Proposition 2.3, we no longer have the doubling condition. However, the growth rate of $\left\|P_{t}^{V}\right\|_{1,1}$ here is worse. On the other hand, the proof, a simple application of the idea of [14], is much shorter. In case $p>2$, the above estimate is essentially contained in [14], see also [25]. We present the proof for completeness.

Case 1. Assume $V \in L^{1}(M)$.

Fixing $y$, we write

$$
u(x, t)=p_{t}^{V}(x, y) .
$$

In this case the proof is almost identical to that of Case 1, Proposition 2.3. The only change is that we use the small time bound (5.1) on $p_{t}^{V}$ and the subexponential volume growth of $M$ (due to Assumption (C)) to conclude that

$$
\int_{M} u(x, 1) d \mu(x)=\int_{M} p_{1}^{V}(x, y) d \mu(x) \leq C .
$$

The rest of the proof is identical.

Case 2. Assume $V \in L^{p}(M, \mu)$ with $1<p<2$.

This is almost identical to that of Case 2, Proposition 2.3. Indeed, from that case, we have

$$
\int_{M} u(x, t) d \mu(x) \leq C+C^{\prime} \sqrt{t}\left(\int_{1}^{t} \int_{M} u^{2}(x, s) d \mu(x) d s\right)^{(p-1) / 2}
$$

Since $p_{t}^{V}$ is contractive in $L^{2}$, this implies

$$
\int_{M} u(x, t) d \mu(x) \leq C t^{p / 2}, \quad t \geq 1 .
$$

Case 3. Assume $V \in L^{p}(M, \mu)$ with $p \geq 2$.

Let $u=u(x, t)$ be as above. Then, as before,

$$
\begin{aligned}
\int_{M} u(x, t) d \mu(x) & \leq \int_{M} u(x, 1) d \mu(x)+\int_{1}^{t} \int_{M} V(x) u(x, s) d \mu(x) d s \\
& \leq C+\int_{1}^{t}\|V\|_{p}\|u(\cdot, s)\|_{p /(p-1)} d s \\
& \leq C+C\|V\|_{p} \int_{1}^{t}\left\|P_{s}^{V}\right\|_{1, p /(p-1)} d s .
\end{aligned}
$$

Applying Riesz-Thorin interpolation with the parameters

$$
p_{2}=1, q_{2}=p /(p-1) ; p_{0}=1, q_{0}=2 ; p_{1}=1, q_{1}=1 ; \theta=\left(2-q_{2}\right) / q_{2},
$$

we have

$$
\frac{1}{p_{2}}=\frac{1-\theta}{p_{0}}+\frac{\theta}{p_{1}}, \frac{1}{q_{2}}=\frac{1-\theta}{q_{0}}+\frac{\theta}{q_{1}}
$$


and

$$
\begin{aligned}
\left\|P_{s}^{V}\right\|_{1, p /(p-1)}=\left\|P_{s}^{V}\right\|_{p_{2}, q_{2}} & \leq\left\|P_{s}^{V}\right\|_{p_{0}, q_{0}}^{1-\theta}\left\|P_{s}^{V}\right\|_{p_{1}, q_{1}}^{\theta} \\
& =\left\|P_{s}^{V}\right\|_{1,2}^{2 / p}\left\|P_{s}^{V}\right\|_{1,1}^{1-(2 / p)} .
\end{aligned}
$$

Therefore,

$$
\int_{M}|u(x, t)| d \mu(x) \leq C+\|V\|_{p} \int_{1}^{t}\left\|P_{s}^{V}\right\|_{1,2}^{2 / p}\left\|P_{s}^{V}\right\|_{1,1}^{1-(2 / p)} d s
$$

Notice that

$$
\left\|P_{s}^{V}\right\|_{1,2} \leq\left\|P_{s}^{V}\right\|_{2, \infty} \leq\left\|P_{s-1}^{V}\right\|_{2,2}\left\|P_{1}^{V}\right\|_{2, \infty} \leq C .
$$

We obtain

$$
\int_{M}|u(x, t)| d \mu(x) \leq C+\|V\|_{p} \int_{1}^{t}\left\|P_{s}^{V}\right\|_{1,1}^{1-(2 / p)} d s
$$

i.e.,

$$
\left\|P_{t}^{V}\right\|_{1,1} \leq C+\|V\|_{p} \int_{1}^{t}\left\|P_{s}^{V}\right\|_{1,1}^{1-(2 / p)} d s .
$$

From here it is easy to see that

$$
\left\|P_{t}^{V}\right\|_{1,1} \leq C t^{p / 2}
$$

This completes Step 1.

Step 2.

Write

$$
I(t)=\int_{M} u^{2}(x, t) d \mu(x) .
$$

As in the proof of Proposition 2.2, the strong positivity of $\Delta-V$ yields

$$
I(t) \leq \frac{\int_{\{x \mid u(x, t)>s\}}|\nabla(u(x, t)-s)|^{2} d \mu(x)}{\lambda_{1}(\{x \mid u(x, t)>s\})}+2 s F(t) .
$$

Here, according to (5.3), $F(t)=t^{p / 2}$.

Using the fact that

$$
|\{x \mid u(x, t)>s\}| \leq s^{-1} \int_{M} u(x, t) d \mu(x),
$$

and $(U F K)$, we deduce

$$
I(t) \leq \frac{\int_{\{x \mid u(x, t)>s\}}|\nabla(u(x, t)-s)|^{2} d \mu(x)}{\Lambda\left(s^{-1} F(t)\right)}+2 s F(t) .
$$

Hence

$$
\int_{\{x \mid u(x, t)>s\}}|\nabla u(x, t)|^{2} d \mu(x) \geq[I(t)-2 s F(t)] \Lambda\left(s^{-1} F(t)\right) .
$$

By the strong positivity of $\Delta-V$, we have as usual

$$
I^{\prime}(t) \leq-2(1-A) \int_{M}|\nabla u(x, t)|^{2} d \mu(x),
$$


thus the combination of the above inequalities yields

$$
I^{\prime}(t) \leq-2(1-A)[I(t)-2 s F(t)] \Lambda\left(s^{-1} F(t)\right) .
$$

Take $s F(t)=I(t) / 4$, i.e.,

$$
s^{-1}=4 I^{-1}(t) F(t) .
$$

Then (5.6) becomes

$$
I^{\prime}(t) \leq-(1-A) I(t) \Lambda\left(4 F^{2}(t) I^{-1}(t)\right) .
$$

Hence

$$
\int_{t}^{2 t} \frac{I^{\prime}(l)}{I(l) \Lambda\left(4 F^{2}(l) I^{-1}(l)\right)} d l \leq-(1-A) t .
$$

Notice that $\Lambda$ is a decreasing and $F$ is an increasing function. Therefore, for $l \geq t$,

$$
\Lambda\left(4 F^{2}(l) I^{-1}(l)\right) \leq \Lambda\left(4 F^{2}(t) I^{-1}(l)\right) .
$$

Consequently,

$$
\int_{t}^{2 t} \frac{I^{\prime}(l)}{I(l) \Lambda\left(4 F^{2}(t) I^{-1}(l)\right)} d l \leq-(1-A) t .
$$

Take $\eta=4 F^{2}(t) I^{-1}(l)$. One gets

$$
\int_{4 F^{2}(t) I^{-1}(t)}^{4 F^{2}(t) I^{-1}(2 t)} \frac{d \eta}{\eta \Lambda(\eta)} \geq(1-A) t .
$$

Following the definition of $\gamma$, i.e., $t=\int_{0}^{\gamma} \frac{d \eta}{\eta \Lambda(\eta)}$, we have

$$
\frac{4 F^{2}(t)}{I(2 t)} \geq \gamma((1-A) t)
$$

i.e.,

$$
I(t) \leq \frac{4 F^{2}(t)}{\gamma(c t)}
$$

From here the desired bound for $p_{t}^{V}$ is a consequence of (5.3), and the bound on $\vec{p}_{t}$ follows as usual by domination.

q.e.d.

Let us conclude by writing a semigroup version of the last part of the proof of Theorem 5.1, in the spirit of [8], where the case $F$ bounded is treated. We leave the details to the reader.

Proposition 5.1. Let $(M, \mu)$ be a $\sigma$-finite measure space, and $T_{t}$ be a semigroup acting on $L^{p}(M, \mu)$, for $1 \leq p \leq+\infty$, with infinitesimal generator $-A$. Suppose that there exists a non-decreasing function $F$ on $\mathbb{R}_{+}$such that

$$
\left\|T_{t}\right\|_{1 \rightarrow 1},\left\|T_{t}\right\|_{\infty \rightarrow \infty} \leq F(t), \forall t>0
$$

and that

$$
\theta\left(\|f\|_{2}^{2}\right) \leq \operatorname{Re}(A f, f), \forall f \in D(A),\|f\|_{1} \leq C,
$$


for some $C>0$, where $\theta:(0,+\infty) \rightarrow(0,+\infty)$ is continuous and satisfies $\int^{+\infty} \frac{d s}{\theta(s)}<+\infty$. Then $T_{t}$ is ultracontractive and

$$
\left\|T_{t}\right\|_{1 \rightarrow \infty} \leq C F^{2}(t) m(C t), \forall t>0,
$$

for some $C>0$, where $m$ is the solution of

$$
-m^{\prime}(t)=\theta(m(t))
$$

on $(0,+\infty)$ such that $m(0)=+\infty$, or alternatively, the inverse function of $p(t)=\int_{t}^{+\infty} \frac{d x}{\theta(x)}$.

\section{References}

[1] P. Auscher, T. Coulhon, X.-T. Duong, \& S. Hofmann, Riesz transform on manifolds and heat kernel regularity, Ann. Sc. E.N.S. 37 (2004) 911-957, MR 2185868, Zbl 1086.58013.

[2] I. Benjamini, I. Chavel, \& E. Feldman, Heat kernel lower bounds on manifolds using the old ideas of Nash, Proc. London Math. Soc. 72 (1996) 215-240, MR 1357093, Zbl 0853.58098.

[3] N. Berline, E. Getzler, \& M. Vergne, Heat kernels and Dirac operators. SpringerVerlag, 1992, MR 2273508, Zbl 1037.58015.

[4] G. Carron, Inégalités de Hardy sur les variétés riemanniennes non-compactes, J. Math. Pures Appl. (9) 76(10) (1997) 883-891, MR 1489943, Zbl 0886.58111.

[5] N. Charalambous, On the $L^{p}$ independence of the spectrum of the Hodge Laplacian on non-compact manifolds, J. Funct. Anal. 225 (2005) 22-48, MR 2139103, Zbl 1083.58026 .

[6] J. Cheeger, M. Gromov, \& M. Taylor, Finite propagation speed, kernel estimates for functions of the Laplace operator, and the geometry of complete Riemannian manifolds, J. Differential Geom. 17(1) (1982) 15-53, MR 0658471, Zbl 0493.53035.

[7] S.Y. Cheng \& S.-T. Yau, Differential equations on Riemannian manifolds and their geometric applications, Comm. Pure Appl. Math. 28(3) (1975) 333-354, MR 0385749, Zbl 0312.53031.

[8] T. Coulhon, Ultracontractivity and Nash type inequalities, J. Funct. Anal. 141(2) (1996) 510-539, MR 1418518, Zbl 0887.58009.

[9] _ Heat kernel and isoperimetry on non-compact Riemannian manifolds, in 'Heat kernels and analysis on manifolds, graphs and metric spaces' (P. Auscher, T. Coulhon, A. Grigor'yan, eds.), AMS, Contemporary Mathematics 338 (2004) 65-99, MR 2039952, Zbl 1045.58016.

[10] T. Coulhon \& X.T. Duong, Riesz transforms for $1 \leq p \leq 2$, Trans. AMS 351 (1999) 1151-1169, MR 1458299, Zbl 0973.58018.

[11] _ Riesz transform and related inequalities on non-compact Riemannian manifolds, Comm. on Pure and Applied Math. 56(12) (2003) 1728-1751, MR 2001444, Zbl 1037.58017.

[12] T. Coulhon \& A. Grigor'yan, On-diagonal lower bounds for heat kernels and Markov chains, Duke Math. J. 89(1) (1997) 133-199, MR 1458975, Zbl 0920.58064 . 
[13] E.B. Davies, Heat kernel bounds, conservation of probability and the Feller property, J. Anal. Math. 58 (1992) 99-119, MR 1226938, Zbl 0808.58041.

[14] E.B. Davies \& B. Simon, $L^{p}$ norms of noncritical Schrödinger semigroups, J. Funct. Anal. 102(1) (1991) 95-115, MR 1138839, Zbl 0743.47047.

[15] H. Donnelly \& P. Li, Lower bounds for the eigenvalues of Riemannian manifolds, Michigan Math. J. 29(2) (1982) 149-161, MR 0654476, Zbl 0488.58022.

[16] S. Gallot \& D. Meyer, Opérateur de courbure et laplacien des formes différentielles d'une variété riemannienne, J. Math. Pures Appl. (9) 54(3) (1975) 259-284, MR 0454884, Zbl 0316.53036.

[17] A. Grigor'yan, The heat equation on noncompact Riemannian manifolds (Russian), Mat. Sb. 182(1) (1991) 55-87; (English translation) Math. USSR Sb. 72 (1992) 47-77, MR 1098839, Zbl 0776.58035.

[18] - Heat kernel upper bounds on a complete non-compact manifold, Rev. Mat. Iberoamer. 10(2) (1994) 395-452, MR 1286481, Zbl 0810.58040.

[19] _ Integral maximum principle and its applications, Proc. Roy. Soc. Edinburgh 124A (1994) 353-362, MR 1273753, Zbl 0812.58082.

[20] _ Gaussian upper bounds for the heat kernel on arbitrary manifolds, J. Differential Geom. 45(1) (1997) 33-52, MR 1443330, Zbl 0865.58042.

[21] A. Grigor'yan \& S.-T. Yau, Isoperimetric properties of higher eigenvalues of elliptic operators, Amer. J. Math. 125 (2003) 893-940, MR 1993744, Zbl 1042.58017 .

[22] H. Hess, R. Schrader, \& D.A. Uhlenbrock, Domination of semigroupsand generalization of Kato's inequality, Duke Math. J. 44(4) (1977) 893-904, MR 0458243, Zbl 0379.47028.

[23] Kato's inequality and the spectral distribution of Laplacians on compact Riemannian manifolds, J. Differential Geom. 15(1) (1980) 27-37, MR 0602436, Zbl 0442.58032.

[24] D. Levin \& M. Solomyak, The Rozenblum-Lieb-Cwikel inequality for Markov generators, J. Anal. Math. 71 (1997) 173-193, MR 1454250, Zbl 0910.47017.

[25] X.D. Li, Riesz transforms for diffusion operators on complete Riemannian manifolds, Rev. Mat. Iberoamericana 22(2) (2006) 591-648, MR 2294791.

[26] P. Li \& S.-T. Yau, On the parabolic kernel of the Schrödinger operator, Acta Math. 156 (1986) 153-201, MR 0834612, Zbl 0611.58045.

[27] N. Lohoué, Estimation des projecteurs de De Rham Hodge de certaines variétés riemanniennes non-compactes, Math. Nachr. 279(3) (2006) 272-298, MR 2200666, Zbl 1095.58006.

[28] E.-M. Ouhabaz, Comportement des noyaux de la chaleur des opérateurs de Schrödinger et applications à certaines équations paraboliques semi-linéaires, J. Funct. Anal. 238(1) (2006) 278-297, MR 2253016, Zbl 1106.58019.

[29] S. Rosenberg, Semigroup domination and vanishing theorems, in 'Geometry of Random motion', R. Durrett et al., eds, Contemporary Math., 73, AMS, 1988, 287-302, MR 0954646, Zbl 0657.53022.

[30] _ The Laplacian on a Riemannian manifold, London Math. Soc. Students Texts, 31, Cambridge U.P., 1997, MR 1462892, Zbl 0868.58074.

[31] M. Rumin, Differential geometry on C-C spaces and application to the NovikovShubin numbers of nilpotent Lie groups, C.R.A.S. Paris, série I 329(11 (1999) 985-990, MR 1733906, Zbl 0982.53029. 
[32] _ Around heat decay on forms and relations of nilpotent Lie groups, in 'Séminaire de Théorie spectrale et géométrie de Grenoble', Vol. 19 (2000-2001) 123-164, MR 1909080, Zbl 1035.58021.

[33] A. Sikora, On-diagonal estimates on Schrödinger semigroup kernels and reduced heat kernels, Comm. Math. Phys. 188(1) (1997) 233-249, MR 1471338, Zbl 0884.35027.

[34] _ Riesz transform, Gaussian bounds and the method of wave equation, Math. Z. 247(3) (2004) 643-662, MR 2114433, Zbl 1066.58014.

[35] B. Simon, Large time behavior of the $L^{p}$ norm of Schrödinger semigroups, J. Funct. Anal. 40(1) (1981) 66-83, MR 0607592, Zbl 0478.47024.

[36] _ Schrödinger semigroups, Bull. AMS 7 (1982) 447-526, MR 0670130, Zbl 0524.35002.

[37] R. Strichartz, Analysis of the Laplacian on the complete Riemannian manifold, J. Funct. Anal. 52 (1983) 48-79, MR 0705991, Zbl 0515.58037.

[38] K.-Th. Sturm, Schrödinger semigroups on manifolds, J. Funct. Anal. 118(2) (1993) 309-350, MR 1250266, Zbl 0804.58053.

[39] M. Takeda, Gaussian bounds of heat kernels for Schrödinger operators on Riemannian manifolds, Bull. Lond. Math. Soc. 39(1) (2007) 85-94, MR 2303523, Zbl 1111.60059 .

[40] Q.S. Zhang, On a parabolic equation with a singular lower order term. II. The Gaussian bounds, Indiana Univ. Math. J. 46(3) (1997) 989-1020, MR 1488344, Zbl 0909.35054.

[41] _ An optimal parabolic estimate and its applications in prescribing scalar curvature on some open manifolds with Ricci $\geq 0$, Math. Ann. 316 (2000) 703731, MR 1758450, Zbl 0974.53031.

[42] — Large time behavior of Schrödinger heat kernels and applications, Comm. Math. Phys. 210(2) (2000) 371-398, MR 1776837, Zbl 0978.35014.

[43] _ Global bounds of Schrödinger heat kernels with negative potentials, J. Funct. Anal. 182(2) (2001) 344-370, MR 1828797, Zbl 0985.58008.

[44] Q.S. Zhang \& Z. Zhao, Estimates of global bounds for some Schrödinger heat kernels on manifolds, Ill. J. Math. 44(3) (2000) 556-573, MR 1772429, Zbl 0985.35016.

DÉpartement de Mathématiques Université de Cergy-Pontoise 95302 Pontoise, France

E-mail address: Thierry.Coulhon@u-cergy.fr

Department of MATHEMATICS UNIVERSity OF CALIFORNIA RIVERside, CA 92521

E-mail address: qizhang@math.ucr.edu 